\title{
罚 New Method Speeds Up Identification of Viruses and Bacteria
}

$T$ The current coronavirus crisis highlights the need for fast and accurate detection of infections. Both viral infections like coronavirus and bacterial infections can be detected by screening for genetic materials (e.g. DNI. but everything below could be applied to RNA as well) in patient samples. But this is challenging because the amount of disease DNA is small and it has to be detected in the presence of other. non-disease. DNI. There is a great need to improve the sensitivity of the current methods and to design simple and reliable ways to detect DNI of interest in the presence of other genetic materials.

The standard approach is to design molecular probes that bind strongly to the disease DNA but not to the nondisease DNA. In the new study computer simulations have been used to demonstrate how this could be done better. The idea is that instead of designing molecular probes that bind strongly to one place on the target DNI. the researchers should. counterintuitively. design probes that bind weakly all over the target DNA and exploit the concept of superselectivity to realize selective multivalent binding of the probes to the target DNA. The research team has developed a numerical scheme to identify optimal probe sequences and tested our approach in large-scale. coarse-grained simulations. The designed probes can indeed distinguish between viral and bacterial genome. and even between two different strands of $E$. coli bacterium.

This work opens up new possibilities to construct robust and cost-efficient methods to detect infectious diseases. Given the urgent need for fast. reliable disease detection methods, it is expected to have a strong impact and to encourage new experimental work leading to development of new health care products.

The study entitled Computational design of probes 10 detect bacterial genomes by multiratent binding was published in the Proceedings of the National Academy of Sciences of the USA. The work coordinated by Rosalind J. Allen was performed within a multinational team of researchers in UK. China and Slovenia, with a crucial involvement of IoP: besides the group members Tine Curk. James D. Farrell and Jure Dobnikar. regular visiting fellows Daan Frenkel. Erika Eiser and Stefano Angioletii- U berti were involved.

\section{Contact}

Institute of Physics, CAS

Jure Dobnikar

Jd489@cam.ac.uk

\section{Reference}

T. Curk, C. A. Brackley, J. D. Farrell, Z. Xing, D. Joshi, S. Direito, U. Bren, S. Angioletti-Uberti, J. Dobnikar, E. Eiser, D. Frenkel, R. J. Allen, Computational design of probes to detect bacterial genomes by multivalent binding, accepted for publication, Proc. Nat. AC. SCI. USA 117 (16) 8719-8726 (2020), https://doi.org/10.1073/pnas.1918274117 